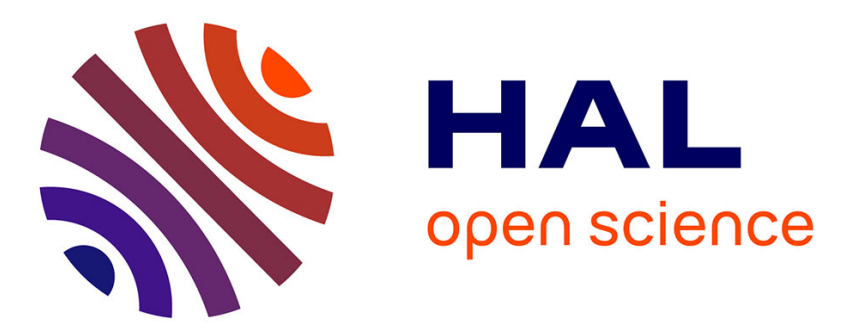

\title{
Lossless Compression Based on a Discrete and Exact Radon Transform: A Preliminary Study
}

Florent Autrusseau, Benoît Parrein, Myriam Servières

\section{To cite this version:}

Florent Autrusseau, Benoît Parrein, Myriam Servières. Lossless Compression Based on a Discrete and Exact Radon Transform: A Preliminary Study. International Conference on Acoustics, Speech and Signal Processing, 2006, Toulouse, France. pp.425-428. hal-00250684

\section{HAL Id: hal-00250684 https://hal.science/hal-00250684}

Submitted on 12 Feb 2008

HAL is a multi-disciplinary open access archive for the deposit and dissemination of scientific research documents, whether they are published or not. The documents may come from teaching and research institutions in France or abroad, or from public or private research centers.
L'archive ouverte pluridisciplinaire HAL, est destinée au dépôt et à la diffusion de documents scientifiques de niveau recherche, publiés ou non, émanant des établissements d'enseignement et de recherche français ou étrangers, des laboratoires publics ou privés. 


\title{
LOSSLESS COMPRESSION BASED ON A DISCRETE AND EXACT RADON TRANSFORM: A PRELIMINARY STUDY
}

\author{
F. Autrusseau, B. Parrein, M. Servières \\ IRCCyN-IVC, École polytechnique de l'Université de Nantes, \\ Rue Christian Pauc, La Chantrerie, \\ 44306 Nantes Cedex 3, FRANCE.
}

\begin{abstract}
This article presents a new lossless compression algorithm based on a Finite Radon Transform. The baseline of this work consist in encoding the difference between several similar finite Radon projections. Two differential encoding techniques are needed to efficiently encode the Radon transformed data. An intra-projection encoding technique is first performed, this differential encoding process takes benefit of the redundancy present in such projections. The second step consists in encoding the similarities between Radon projections, this is the inter-projection encoding procedure. The used Finite Radon transform (called Mojette transform), allows to ensure both compression and data redundancy. The main novelty of this work is the use of a secure distributed storage or transmission tool in a lossless compression context.
\end{abstract}

\section{INTRODUCTION}

We present here a joint source channel coding technique based on a finite Radon transform [R1]. The most popular applications of the Radon transform takes place in medical imaging context. This transform may be used to reconstruct images from medical CT scans. In fact, the Radon transform is widely exploited in the tomography context. A few finite Radon transform exist in the open literature [AGB1, S1]. The version exploited here is named Mojette transform [AGB1], it has been mostly used in a transmission context. Effectively, this Discrete version of the Radon Transform is exactly invertible, and extremely tunable in terms of redundancy. The main applications of the Mojette transform so far were: multiple description coding, Forward Error Correcting Codes or encryption, dedicated to distributed storage area or quality of service network. A few attempts to perform compression techniques based on finite Radon transform have exploited the central slice theorem [MOLP2]. This work had a dual goal, the two intended applications were image compression and pattern detection. The image reconstruction was based on the Fourier central slice theorem. This process strongly differs from the one we will see in the following. The central slice theorem applies a Fourier transform on the Radon projections, and place the so-obtained coefficients in a Fourier Spectrum. Finally, an inverse Fourier transform provides the reconstructed image. In the presented study, the goal is to propose a joint sourcechannel encoding technique, i.e. we have to satisfy the complex task of providing both compression (source coding) and redundancy (channel coding). To the best of our knowledge, no technique have ever combined redundancy and compression by exploiting the same operator (Radon transform). Furthermore, the inverse Radon transform used here is totally different from the central slice theorem. The general principle of this work is to exploit the redundancy provided by the Mojette transform in a differential coding technique. Effectively, an interesting property of the Mojette transform is the periodicity appearing within the projections. Furthermore, by adequately choosing the projection direction set (very close angles), important similarities will inevitably occur between projections. This work exploits both the periodicity within each projection, and the similarities between projections by applying two separate differential coding techniques. Moreover, such process may be exploited in an encryption context. It has been demonstrated that applying the inverse Mojette transform on erroneous bins leads to a quick propagation of errors, providing encrypted images [AGB1]. Such encryption framework is briefly envisioned here, no new encryption technique is proposed, but the Mojette transform's adaptability to encryption algorithms is highlighted by a simple experiment. A unitary incrementation is applied on one single projection, and shows the encrypted reconstructed data.

This paper is decomposed as follows, section 2 will focus on the Mojette transform, both the direct and inverse algorithms will be detailed. The section 3 is devoted to the lossless compression algorithm. We will see how we can take advantage of the similarities between and within projections to efficiently compress the data. Finally, section 4 gives the results for both the compression rate and redundancy coefficient for fifteen grey scale images. 


\section{MOJETTE TRANSFORM}

\subsection{Direct Mojette transform}

The Mojette transform is an exact and discrete Radon transform [R1] defined for specific projection angles. The transformed domain of an image is a set of projections where each element (called a bin like in tomography) corresponds to the sum of the crossed pixels along the projection line. Like the usual Radon transform, the Mojette transform represents the image from a set of projections, but with an exact discrete inverse. The projection angles, provided by a set of vectors $\left(p_{i}, q_{i}\right)$ must respect the condition where $p_{i}$ and $q_{i}$ are prime together. This is a linear transform defined for each projection angle by the operator:

$M_{p, q} f(k, l)=\operatorname{proj}_{p, q}(b)$

$=\sum_{k=-\infty}^{+\infty} f(k, l) \Delta(b+k q-l p)$

where $\Delta(b)=\left\{\begin{array}{l}1 \text { if } b=0 \\ 0 \text { if } b \neq 0\end{array}\right.$, and where the $(k, l)$ pixel belongs to the image. The Mojette transform $M_{I} f(k, l)$ corresponds to the set of $I$ projections $M_{p, q} f(k, l)$ for several angles indexed by $\mathrm{i}$ :

$$
M_{I} f(k, l)=\left\{\operatorname{proj}_{p_{i}, q_{i}}=M_{p_{i}, q_{i}} f, i \in[1 \ldots I]\right\}
$$

Each bin value equals the sum of the pixels crossed by the appropriate line $b=l p-k q$. The main difference with the Radon transform is the sampling on the projections, which depends on the chosen angle. The figure 1 (top panel) represents the Direct Mojette transform according to the directions set $S=\{(0,-1)(1,2)$ and $(-1,1)\}$. The number of bins for each projection depends on the chosen discrete angle. The algorithmic complexity of the Mojette transform for a $N$ pixels region with $I$ projections is $O(I N)$.

\subsection{Inverse Mojette Transform and reconstructibility notion}

The first result on the reconstruction conditions came from Katz [K1] in a different context. An image is reconstructible by a set $I$ of projections when $f(k, l)$ can be obtained only from these $I$ projections. The results found in Katz can be written as the equivalence of two propositions:

i) $f(k, l)$ defined on a rectangular region $G$ (of size $P \times$ $Q)$, can be reconstructed with the set $M_{I} f(k, l)$

\section{ii) $P \leq P_{I}=\sum_{i=1}^{I}\left|p_{i}\right| \quad$ or $\quad Q \leq Q_{I}=\sum_{i=1}^{I}\left|q_{i}\right|$}

Theorem 1. [GN1] The $R$ region is the smallest region being not reconstructible i.e. any region which does not entirely contains $R$ can be reconstructed with $S_{I}$. Another way to describe this result is the equivalence between these two propositions:

i) $f(k, l)$ defined on a convex $G$ can be reconstructed with the set $S_{I}$,

ii) $R$ constructed by dilations with $S_{I}$ cannot be included into $G$.

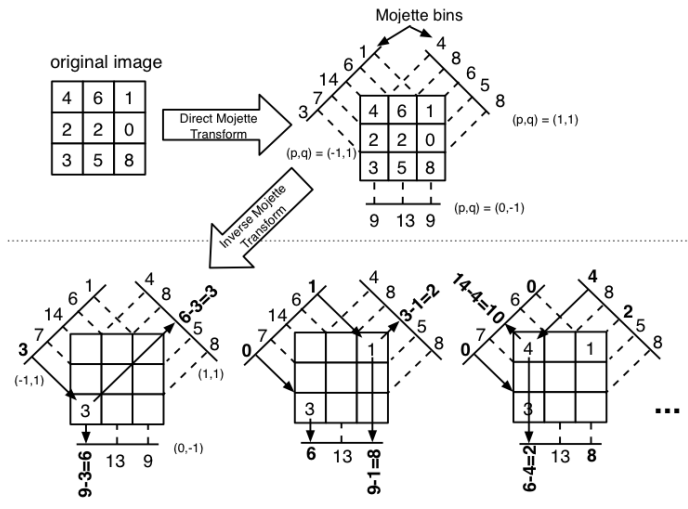

Fig. 1. Direct Mojette Transform (top panel), and the three first steps of the inverse transform (bottom panel).

More details on both the Katz lemma and the reconstructibility notion given by the first theorem can be found in [AGB1]. The inverse Mojette transform corresponds to a simple and fast algorithm. The bin value is back-projected into the pixel and subtracted from all projections. The bottom panel of figure 1 shows a example of the three first steps of the inverse Mojette transform.

\section{COMPRESSION TECHNIQUE}

The proposed compression technique takes advantage of the similarities within each Mojette projection, as well as the similarities between projections. From now on, these two separate techniques will be named "intra-projection coding" and "inter-projections coding". The figure 2 shows the projections of image "watch" (see fig. 5), with 3 direction angles: $\left(p_{i}, q_{i}\right)=(1,128),(1,129),(1,130)$. This figure clearly shows the strong similarities between all projections. The $\mathrm{x}$-axis represents the bins positions, whereas the $y$-axis stands for the bins values (the sum of the crossed pixels). These similarities are even more evident in figure 3, where a zoom is performed. Such strong similarities implies that a very efficient differential coding technique might be exploited. As explained in section 2, the number of bins
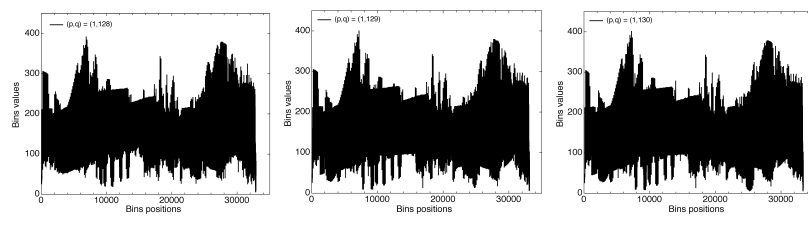

Fig. 2. Mojette projections for directions $(1,128),(1,129)$ and $(1,130)$.

for each projection depends on the chosen direction angle. 
We then have to perform a projection scaling in order to be able to compute the differences projections on the same support size. Evidently, this scaling must be reversible and the chosen support will have to be the biggest one (projection $(1,130)$ in figure 2).

\subsection{Intra-projection coding}

Figure 3 highlights both the intra-projection periodicity and inter-projections similarities. It represents a zoom in of figure 2 for the range [11000, 11400]. The two top figures, and the bottom left one represent a zoom on projections $(1,128)$, $(1,129)$ and $(1,130)$ respectively, while the bottom right figure stands for the intra-projection coding (performed on projection $(1,130))$. The periodicity within the Mojette projec-
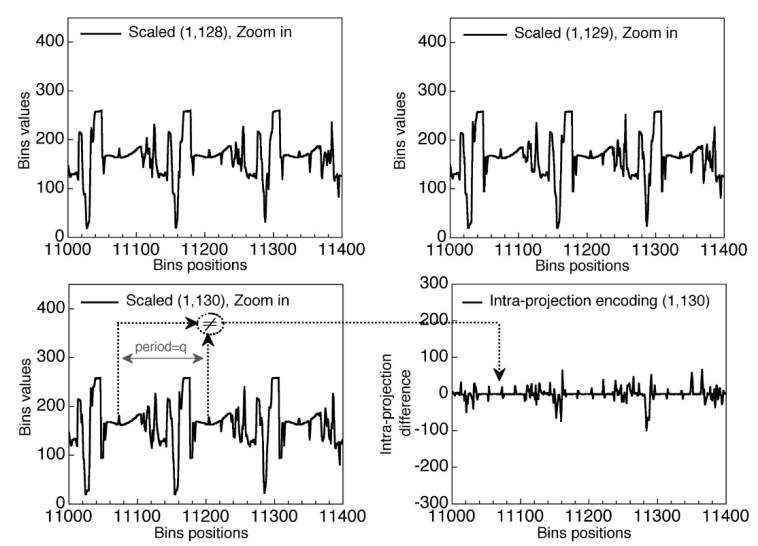

Fig. 3. Zoom in, projections $(1,128),(1,129),(1,130)$, and intra-projection difference (1,130). Range: [11000, 11400].

tions can be efficiently encoded by computing for each bin position, the difference between the current bin value and the value of the bin shifted by one period (this period equals q). This intra-projection encoding principle is depicted by the grey dashed lines in figure 3.

\subsection{Inter-projections coding}

Once the intra-projection coding applied, the inter-projection encoding technique can then be performed. This latter simply computes for each bin position, the pair by pair differences between all projections. For the example given above (figure 3), the inter-projection difference is given in figure 4 The so-obtained difference projections having many values close to zero, we expect an entropy coding technique to be quite efficient on such data. The next section is devoted to the evaluation of the presented technique. Compression rates and redundancy coefficients are given for a set of 15 test images.
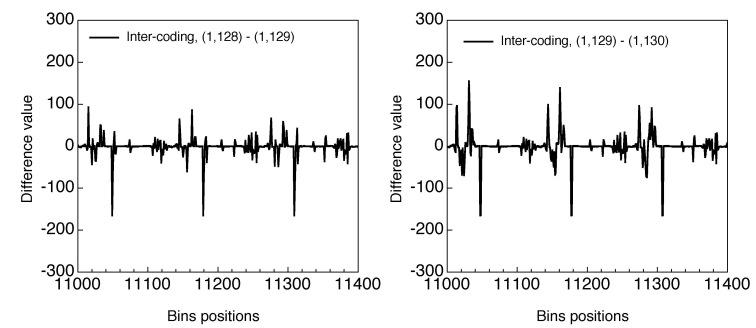

Fig. 4. Inter-projection difference.

\section{RESULTS}

The lossless compression technique have been tested on 15 images for two distinct projections sets. These projection sets are respectively denoted as $S 1$ and $S 2$ :

$S 1=\{(1,128)(1,129)(1,130)\}$ and

$S 2=\{(1,85)(1,86)(1,87)(1,88)\}$. This section presents the results for fifteen grey level images (see figure 5) $(256 \times 256,8$ bits per pixel). Evidently, one could use the same process on colour images, and furthermore, an inter chromaticity-component differential coding could be applied. This will be the focus of a later study. Based on
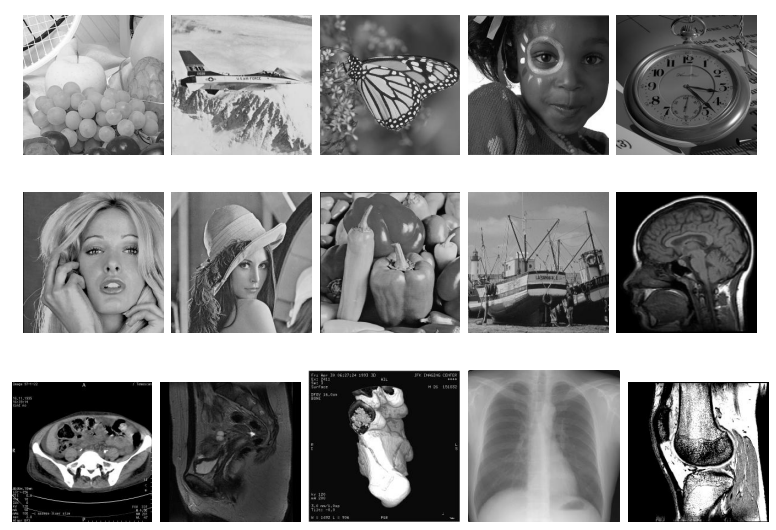

Fig. 5. The fifteen tested images.

this proposed compression technique, at least two options are available for the encoding process. The first choice (denoted as Exp1 in the following) is to apply the intraencoding technique on each projection, and then to perform the inter-encoding process between all intra-encoded projections. This implies that we need to transmit a basis intraencoding projection along with all others inter-encoded ones. The second option (denoted as Exp2) is to compute an overall mean projection (for which each bin equals the mean value of all projections), to apply the intra-encoding technique on this mean projection, and finally compute the interencoding process between all projections and this mean one. 
Table 1 shows the detailed results for Exp1: an intra-encoded projection is transmitted along with the inter-projections differences. Direction set S1 is tested with the "peppers" image.

Table 1. Entropy results for Exp1, direction set S1.

\begin{tabular}{lllll}
\hline peppers, R=1.51 & length & Entropy & Red./ Compr. & Size $(\mathrm{kb})$ \\
\hline Original & $256^{2}$ & & & 65,5 \\
Encoded & $256^{2}$ & 7,5 & $93 \%$ & 61,4 \\
\hline intra-proj & 33406 & 5,81 & & 24,2 \\
inter:(128-129) & 33406 & 5,00 & & 20,8 \\
inter:(129-130) & 33406 & 5,20 & & 21,7 \\
\hline Sum 2 projs & 66812 & & 1,$00 ; 68 \%$ & 45,0 \\
Sum 3 projs & 100218 & & 1,$51 ; 101 \%$ & 66,7 \\
\hline
\end{tabular}

The figure 6 gives both the compression rates (right panel) and overall entropy (left panel) for the whole set of images and for the two experiments. A comparison with lossless JPEG 2000 rates is given in the first panel. As previously mentioned, an encryption algorithm can easily be derived. An encryption algorithm on the "basis" projection will automatically lead to decode erroneous bins (during the differential decoding), and thus, to back-project errors into the image. A former study [AGB1] demonstrated the very quick and devastating propagation of such errors into the reconstructed image. Two encrypted images are given in figure 7 (each bin simply underwent a unitary incrementation).
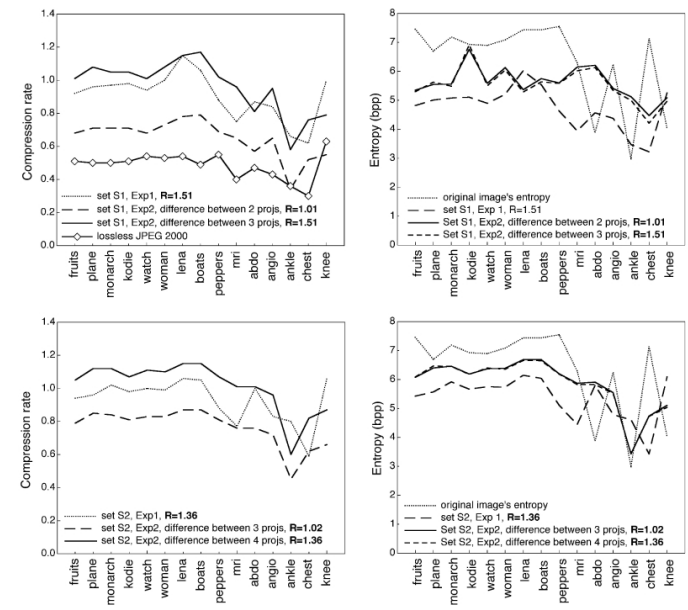

Fig. 6. Compression rates (top panel) and entropy (bottom panel) for 15 images and for 2 direction sets.

\section{CONCLUSIONS}

This work presents a new lossless encoding technique based on a Finite Radon Transform. The aim of this preliminary

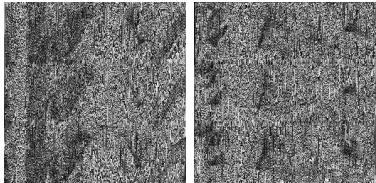

Fig. 7. Encrypted images "lena" and "peppers", only one modified projection (by simple unitary incrementation).

study was to introduce the idea of inter- and intra-projection differential coding. One could evidently have chosen to first apply a standard compression algorithm to the image, and to compute the Mojette projections afterwards, on the compressed data. Nevertheless, the idea of merging the data into one important projection can be efficiently exploited by encryption algorithms. Furthermore, we are convinced that an optimisation of both the intra and inter projection differences may provide much higher compression rates. This will indeed be the main focus of further researchs. Even though the results so far can not compete with usual joint source-channel encoding techniques, we believe that many improvements could be performed to sensibly improve the ratio between the compression rate and redundancy. For instance, a Run Length Encoding (RLE) technique might be very helpful on the inter-projection encoding results.

\section{REFERENCES}

[K1] M. Katz, "Questions of uniqueness and resolution in reconstruction from projections", Lect. Notes in Biomath., Springer Verlag, 1997.

[R1] J. Radon, "Uber die bestimmung von functionen durch ihre integralwerte langs gewisser mannigfaltigkeiten", Berichte Sachsische Academie der Wissenchaften, (69), 262-267, 1917.

[S1] I. Svalbe, "Linear and Non-linear Image Processing Operations on Digital Projections", Intel. Symp. on Math. Morphology VI, 2002.

[AGB1] F. Autrusseau, JP Guédon, Y. Bizais, "Watermarking and cryptographic schemes for medical imaging”, Med. Imaging, 5032-105, 2003.

[GN1] JP. Guédon, N. Normand, "The Mojette Transform : The First Ten Years”, DGCI, 79-91, 2005.

[MOLP2] E. Magli, G. Olmo, L. LoPresti, "Selection and compression of images of interest based on the Radon transform", Recent Research Developments in Pattern Recognition, Vol. 1, 2000. 